\title{
Cluster analysis of top 200 universities in Mathematics
}

\begin{abstract}
University rankings are becoming a vital performance assessment for higher learning institutions worldwide. Besides the overall rankings, the universities are also ranked by subjects serving as comprehensive guide to discover the specialist strengths of universities worldwide by highlighting top 200 universities for a range of 30 individual popular subjects. Data for this ranking purpose consist four variables namely the academic reputation, employer reputation, citation per paper and $\mathrm{H}$-index citations. In this ranking, universities are ranked according to their overall score calculated from linear combination of the aforementioned variables and their respective weightings. As the existing ranking technique based on overall score appears to be simple and since the rankings data are of multivariate nature, therefore it is possible to use multivariate statistical technique like cluster analysis. Agglomerative hierarchical cluster analysis of top 200 QS ranked universities by Mathematics subject area 2014 has been performed to obtain natural clustering of the universities in an objective manner. The agreement between cluster analysis and existing QS rankings is verified and it is suggested that the distance between universities can be used as an alternative measure to rank universities. Cluster analysis applied on the same variables would serve as an alternative way to rank universities and to look at the rankings in a different perspective.
\end{abstract}

Keyword: Hierarchical cluster analysis; QS World University Rankings; Rankings by Mathematics subject 